\title{
La crisis de la prensa escrita: una revisión bibliográfica para repensarla desde Latinoamérica
}

\section{The crisis of the written press: A bibliographical review to rethink it from Latin America}

\section{A crise da imprensa escrita: uma revisão bibliográfica para repensá-la a partir da visão da América Latina}

Facundo Suenzo, Northwestern University, Evanston, Estados Unidos (Facundo@u.northwestern.edu)

PabloJ. Boczkowski, Northwestern University, Evanston, Estados Unidos (pjb9@northwestern.edu)

Eugenia Mitchelstein, Universidad de San Andrés, Buenos Aires, Argentina (emitchelstein@udesa.edu.ar)

RESUMEN | En este artículo efectuamos un análisis crítico de 132 publicaciones recientes sobre la crisis de la prensa escrita. Encontramos que la discusión sobre el fenómeno se ha organizado principalmente en torno a tres grupos temáticos: la dimensión económica y financiera para entender la crisis, la descripción y el análisis de las dinámicas laborales de los periodistas, y el posible impacto de la crisis en el rol social y los valores de la prensa. Las investigaciones han sido heterogéneas en cuanto a sus enfoques conceptuales y al conjunto de técnicas utilizadas: fundamentalmente estudios de caso y, en menor medida, estudios comparativos. Además, se han centrado principalmente en las dimensiones productivas de la información, dejando mayormente de lado la recepción de las noticias y el consumo de diario en papel. Finalmente, en el artículo esbozamos un marco posible para repensar la crisis, pero también una agenda de investigación futura, desde y en Latinoamérica.

PALABRAS CLAVE: prensa escrita; diarios en papel; consumo de noticias; consumo de medios; periodismo; crisis de la prensa escrita. 
ABSTRACT | In this article we provide a critical analysis of 132 recent publications on the crisis of the written press. We find that the discussion of the phenomenon has been organized mainly in three thematic groups: the economic and financial dimension to understand the crisis, the description and analysis of the labor dynamics of journalists, and the crisis' possible impact on the press' social role and values. Research has been heterogeneous in terms of their conceptual approaches and the set of techniques used: mostly case studies and - to a lesser extent-comparative studies. Moreover, they have mainly focused on the productive dimensions of information, leaving mostly aside the reception of news and the consumption of newspapers. Finally, we outline a possible framework for rethinking the crisis in this article, but also a future research agenda, from and in Latin America.

KEYWORDS: written press; newspapers; news consumption; media consumption; journalism; crisis of written press.

RESUMO| Neste artigo apresentamos uma análise crítica de 132 publicações recentes sobre a crise da imprensa escrita. Verificamos que a discussão do fenômeno foi organizada principalmente em três grupos temáticos: a dimensão econômica e financeira para a compreensão da crise; a descrição e análise da dinâmica laboral dos jornalistas; e o possível impacto da crise sobre o papel social, e os valores da imprensa. A investigação tem sido heterogênea em termos das suas abordagens conceptuais e do conjunto de técnicas utilizadas: principalmente estudos de caso e, em menor grau, estudos comparativos. Além disso, concentraram-se principalmente nas dimensões produtivas da informação, deixando de lado sobretudo a recepção das notícias e o consumo de jornais de papel. Finalmente, no artigo esboçamos um possível quadro para repensar a crise, mas também uma agenda de investigação futura, desde e na América Latina.

PALAVRAS CHAVE: imprensa escrita; jornais de papel; consumo de notícias; consumo da mídia; jornalismo; crise da imprensa escrita. 


\section{INTRODUCCIÓN}

El rol de los diarios en las sociedades contemporáneas ha sido ampliamente descrito. La prensa supo conformar las representaciones materiales de la esfera pública (Habermas, 2015) y tener un vínculo fundamental en lo que Anderson (2006) definió como identidades nacionales. Sin embargo, en las últimas décadas, los diarios impresos han sufrido caídas dramáticas en los números de lectores e ingresos, así como una reducción en el personal y en los recursos. En Latinoamérica son varios los títulos cuya circulación se ha visto afectada, llevando a algunos a su completa desaparición. En Venezuela, por ejemplo, el diario El Nacional canceló su edición impresa en diciembre de 2018 luego de 75 años de circulación. Otros diarios se han visto fuertemente tocados por la crisis, reduciendo drásticamente sus equipos. Tal es el caso del diario de Pernambuco, uno de los más antiguos en circulación en Brasil, que achicó su redacción en un tercio (Knight Center for Journalism in the Americas, 2018). Esta no fue la suerte del Jornal do Commercio, el diario sobre economía y negocios más antiguo en Río de Janeiro, que -con casi 190 años de existencia- en 2016 cesó sus actividades no solo en su versión impresa, sino también en su portal de Internet (Víctima de la crisis..., 2016). En Argentina, luego de 140 años de circulación, en 2018 cerró el diario en inglés más antiguo de América Latina, The Buenos Aires Herald. Sin embargo, aquellos que todavía persisten también están sufriendo un gran impacto en su circulación. Particularmente, entre 2006 y 2016, Clarín, el diario de mayor circulación en Argentina, perdió casi la mitad de su circulación neta diaria, y su principal competidor, La Nación, más de un quinto (SINCA, n.d.).

La academia no ha sido ajena a este fenómeno global y se ha propuesto documentarlo y explicarlo; si bien han sido muy relevantes, las investigaciones sobre este tema han girado mayormente en torno a las dinámicas de producción de la noticia y los medios.

En primer lugar, los investigadores han abordado la crisis desde su dinámica económica y financiera. Se han encargado de describir los nuevos y viejos modelos de negocio, sus estructuras productivas y cómo mejorar sus eficiencias y rendimientos, así como el impacto de estos nuevos modelos en el periodismo (Retegui, 2013; Schlesinger \& Doyle, 2015).

En segundo lugar, las investigaciones se han ocupado de contextualizar la crisis en los vínculos laborales y la dinámica ocupacional del periodismo. Han indagado en cómo la presión económica y la precarización laboral puede llevar a sentimientos de inseguridad constante en la profesión, y cómo dicha presión pone en cuestión valores tradicionales como la autonomía en el periodismo. En otras palabras, cómo estos nuevos modelos de negocio colaboran en la construcción en una nueva subjetividad 
ocupacional (Bachmann \& Harlow, 2012; Davidson \& Meyers, 2016; Meyers \& Davidson, 2014, 2016; Sarrimo, 2017; Siapera, Papadopoulou, \& Archontakis, 2015).

En tercer lugar, pero en menor medida, el declive de la prensa escrita también impulsó el análisis sobre cómo la caída en la circulación y el consumo del diario en papel, por un lado, y la aparición de nuevas plataformas de contenido e información, por el otro, pueden impactar en el rol de la prensa en las sociedades contemporáneas y democráticas. Las investigaciones han abordado particularmente la crisis de los diarios desde las dinámicas institucionales, la propiedad de los medios y la percepción de sus valores y expectativas, como la responsabilidad y la confianza pública (Conboy \& Eldridge, 2014; Cornia, 2016; Hassid \& Repnikova, 2016; Mor \& Reich, 2018; Porto, 2010; Schlesinger \& Doyle, 2015). En menor medida, también se ha examinado el vínculo entre los periodistas, el público y la recepción mediática en este contexto de crisis (Boczkowski, Mitchelstein, \& Suenzo, 2019; Carlson \& Usher, 2016; Robledo-Dioses \& Atarama-Rojas, 2018; Thurman, 2018; Thurman \& Fletcher, 2019).

Sibien estas tres dimensiones - que luego se describirán con mayor detenimientohan sido extremadamente fructíferas en dar cuenta de las múltiples dimensiones y pliegues de la crisis del diario en papel, han estado principalmente enfocadas en el hemisferio norte, con algunas muy valoradas excepciones (Waisbord, 2017; Schmitz Weiss, de Macedo Higgins Joyce, Harlow, \& Alves, 2018; Retegui, 2020), dejando abierta la oportunidad para explorar los motivos y particularidades del fenómeno en Latinoamérica. A partir de ello, este trabajo tiene dos objetivos; primero, el de organizar y sistematizar cómo los y las investigadores han comprendido y abordado la crisis de la prensa escrita en los últimos años, sus perspectivas y limitaciones, y en segundo lugar, delinear las oportunidades y ofrecer un marco y agenda posible para abordar la problemática desde América Latina.

Para alcanzar dichos objetivos se desplegó una metodología de revisión bibliográfica (Gómez-Luna, Fernando-Navas, Aponte-Mayor, \& BetancourtBuitrago, 2014) en donde se priorizaron, para la búsqueda y análisis, las publicaciones posteriores a la revisión realizada por Siles y Boczkowski (2012). Posteriormente, se procedió a la recolección de la información con diferentes ecuaciones búsqueda ${ }^{1}$, lo que arrojó un total de 132 referencias.

1. Se utilizaron las siguientes combinaciones: "crisis AND diario en papel"; "crisis AND prensa"; "crisis AND periodismo", combinadas con otros vocablos comunes como "caída", "transición", "consumo" utilizando tanto el español como sus posibles variantes en inglés. Para este trabajo se consideraron solamente las publicaciones académicas en revistas dejando de lado actas de congresos, tesis y otros documentos. 
El artículo continúa del siguiente modo: en la primera sección se despliegan las tres dimensiones que tematizan la crisis - económica, laboral y social-, para luego describir los enfoques teóricos y metodológicos que estas investigaciones han impulsado y finalmente elaborar algunas futuras líneas de análisis para pensar el objeto desde y en América Latina.

\section{LAS DIMENSIONES DE LA CRISIS}

En el contexto que diferentes investigadores llaman crisis del diario en papel (Siles \& Boczkowski, 2012), la literatura sobre el tema se ha enfocado en comprender y explicar principalmente qué sucede en las condiciones de producción (Verón, 2013) de la industria y, en menor medida, qué ocurre con las prácticas de consumo y las dinámicas de reconocimiento. Las investigaciones sobre este tema relevadas recientemente han girado en torno a las siguientes áreas de análisis: dinámica económica y financiera, trabajo y lógicas ocupacionales de la profesión, el impacto social y la recepción de la información.

\section{La dimensión económica de la crisis}

El análisis sobre los factores económicos que componen la crisis ha oscilado entre aquellos que impactan en la desaparición definitiva de los diarios y los que posibilitan su resistencia. Para Kolo, "dado los costos para los editores de diarios en papel, los ingresos generados por los medios digitales sobre la base del contenido editorial de la marca, probablemente no se compensen. A largo plazo, el modelo de negocio del editor tradicional está condenado" (2016, p. 14). Ese modelo de negocio, orientado a un "espiral de la publicidad y la circulación", es para Rothmann \& Koch (2014, p.72) una de las razones por las cuales los medios aún no han podido monetizar sus sitios en línea. En ese sentido, Van der Burg y Van den Bulck indican que la supervivencia de estos medios se debe principalmente a la capacidad que tengan para controlar los costos: "a medida que la demanda (es decir, el número de lectores) sigue disminuyendo, los editores de diarios tendrán que reducir aún más sus organizaciones para nivelar los costos a los ingresos" (2017, p. 107). No obstante, Waisbord argumenta que la crisis económica de los diarios en América Latina tiene otros ribetes:

El modelo económico de la prensa comercial en la región nunca estuvo únicamente basado en la publicidad comercial, como el caso de Estados Unidos y gran parte de Europa Occidental. Fondos públicos administrados por el Estado como así también los intereses corporativos de propietarios cumplieron un papel preponderante en el financiamiento de la prensa comercial. Acuerdos políticos-económicos con quien detentara el poder público y subsidios cruzados de la propia empresa fueron parte central del trípode financiero que generalmente mantuvo al periodismo (2017, p. 21). 
Varios estudios buscaron explicar cómo la crisis afectó principalmente a los modelos de negocio y a las estructuras de producción (Brandstetter \& Schmalhofer, 2014; Doyle, 2013; Gómez \& Louzada, 2017; Ihlström Eriksson, Akesson, \& Lund, 2016; Picard, 2014; Rothmann \& Koch, 2014; Schlesinger \& Doyle, 2015) y sus impactos financieros (Angelucci \& Cagé, 2016; Godoy \& Ospina, 2014; van der Burg \& Van den Bulck, 2017). En un ecosistema de medios catalogado como ubicuo (Ihlström Eriksson et al., 2016), hay quienes describen este período como de reinvención de los medios a través de negocios multiplataforma (Doyle, 2013). Para Doyle "[lo] característico de este cambio es la forma en que las decisiones de contenido se están configurando cada vez más por el potencial para generar valor y rendimientos para el consumidor a través de la distribución de ese contenido en multiplataformas y, también, el papel influyente que desempeñan los datos de la ruta de retorno digital" (2013, p. 17). Siguiendo el concepto de destrucción creativa de Schumpeter, Schlesinger y Doyle (2015), describen cómo el cambio a digital en el Financial Times y The Telegraph tuvo importantes consecuencias para la organización de la producción, argumentando que el uso de la analítica de datos (data analytics) dio nuevas formas a las historias que se cuentan. Como expresó un analista financiero del Financial Times: "Puedo ver a través de nuestros análisis exactamente lo que... las personas están haciendo en el sitio y en realidad estoy entendiendo su comportamiento y sus necesidades... y juzgo su comportamiento en cuanto a qué partes podemos mejorar, y que lleva a la innovación de productos" (Schlesinger \& Doyle, 2015, p. 9). Sin embargo, para los autores, las rutinas y valores tradicionales asociados con la producción del diario impreso continúan ejerciendo una fuerte influencia (Schlesinger \& Doyle, 2015).

A partir de los cambios en los modelos de negocio, los investigadores también examinaron las implicancias en el contenido de las noticias y los medios (Beckers et al., 2017; Brandstetter \& Schmalhofer, 2014; De La Torre \& Dillon, 2012; García Rubio, 2013; Hofstetter \& Schoenhagen, 2017). Brandstetter y Schmalhofer (2014) analizaron la implementación del muro pago en el diario Alemán Die Welt y encontraron que no había una estrategia de contenido diferenciada ni "un mayor uso de las posibilidades que ofrece Internet: no utiliza(n) la multimedialidad, la hipertextualidad (ni) la interactividad" (2014, p. 505). Los hallazgos de Bachmann y Harlow (2012) se encuentran en sintonía. Para las investigadoras, en 2012, solo algunos diarios latinoamericanos habían sacado ventaja del potencial multimedia e interactivo de Internet:

las fotos fueron publicadas en casi todas las historias, pero videos, audios, presentaciones animadas, e infografías fueron incluidas en menos del diez por ciento de las historias analizadas. Además, menos de un quinto de los artículos incluyeron hipervínculos dentro del texto de la historia, y menos de la mitad incluyó hipervínculos a historias relacionadas (2012, p. 227). 
En ese sentido, los imperativos comerciales, junto con las normas del periodismo web caracterizadas principalmente por la velocidad, hacen que "en muchas ocasiones el contenido se replique o acorte entre los diferentes canales de comunicación de los diarios" (Barredo-Ibáñez et al., 2017, p. 16) mostrando un “comportamiento cuasiinteractivo" (Barredo-Ibáñez et al., 2017, p. 287), en el que prevalecen las estrategias residuales de la prensa tradicional. Sorprendentemente, aún en el contexto de crisis, los hallazgos de Beckers y sus colegas (2017) indican que no parece haber un impacto negativo en la diversidad del contenido a lo largo del tiempo. Estos hallazgos coinciden con los de Retegui, aunque para la autora:

\begin{abstract}
El problema no es el sistema de métricas per se (...) Lo que genera tensión entre los periodistas es el choque entre la lógica basada exclusivamente en lo que dictan los algoritmos y aquella basada en los criterios noticiables. Esto está relacionado con la cultura profesional de las redacciones, con las características de las industrias culturales (producir noticias no es producir en serie, como en otros sectores económicos) y con la tendencia a evaluar en términos cuantificables la profesión del periodista, centrada en el trabajo creativo, aun en la era de los datos (2020, p. 62).
\end{abstract}

En esta tensión entre la tradición y el cambio (Mitchelstein \& Boczkowski, 2009, p. 575), también se encuentran rechazos hacia la transformación digital e intentos de mantener la industria del diario impreso lo más intacta posible. Godoy y Ospina (2014) revisaron el mercado de ejemplares impresos en Colombia y encontraron que, entre 2006 y 2012, varios indicadores de eficiencia financiera y económica mostraron signos positivos, como crecimiento en las ventas. Otra razón para explicar el apego al modelo tradicional puede sintetizarse en el valor simbólico del diario frente a otros competidores digitales. Como sostienen Villi y Hayashi:

La impresión en papel también es una ventaja y un símbolo para los periódicos japoneses, especialmente contra los gigantes en línea globales como Google, Yahoo y Facebook que son puramente digitales en su oferta de contenido. La impresión es lo único con lo que los periódicos pueden identificarse (2017, p. 972).

En conclusión, la dimensión económica de la crisis interpela a las organizaciones de noticias para replantearse su estructura interna, para comprender y dimensionar nuevos modelos de negocio y estructuras financieras. Las nuevas estrategias impactan no solo internamente sino también en el contenido que se ofrece a los lectores. Sin embargo, las decisiones están marcadas por un período de transición que todavía muestra alto nivel de rechazo a la tecnología y, por lo tanto, el rechazo a aceptar que el modelo tradicional del papel pueda desaparecer. 


\section{Las manifestaciones laborales de la crisis}

En lo que respecta a las cuestiones laborales, algunos investigadores han examinado cómo los valores profesionales de los periodistas podrían verse afectados por una presunta pérdida de autonomía y condiciones de trabajo precarias debido al control administrativo y la presión económica (Bardan, 2015; Evans, 2016; Goyanes \& Rodríguez-Castro, 2018; Hofstetter \& Schoenhagen, 2017; Reich, 2018; Sarrimo, 2017). En América Latina, donde el periodismo ha sido vulnerable a intereses externos, con lógicas mercantiles y partidarias, "el proyecto profesionalizante, más allá de sus soportes y logros, se encuentra en crisis" (Waisbord 2017, p. 24). Para Sarrimo (2017) hay un cambio de paradigma que hace del periodismo institucionalizado una "industria de empaque" regida por la "lógica industrial y la racionalidad económica" (2017, p. 6). La "industrialización” de la noticia hace que los "periodistas pierdan autonomía subsumiéndose a formatos, módulos y guías de escritura readymade" (2017, p. 6). Hofstetter y Schoenhagen (2017) encontraron que, incluso cuando la integración espacial del equipo editorial mejora la cooperación y las sinergias en diferentes áreas, los imperativos comerciales tienen un impacto negativo en el potencial creativo de producción de medios y contenidos de calidad. Como reacción a la crisis, algunos periodistas "optan por abandonar la profesión, otros se transforman por necesidad y no por elección como periodistas de tiempo parcial o freelancers" (Bardan, 2015, p. 35).

En paralelo, otros estudios han analizado cómo la prevalencia de despidos y cierres en salas de redacción ha provocado un sentimiento de inseguridad laboral e insatisfacción entre los periodistas (Cohen, 2015; Davidson \& Meyers, 2016; Meyers \& Davidson, 2014, 2016; O’Donnell, Zion, \& Sherwood, 2016; Sarrimo, 2017; Siapera et al., 2015). Para Meyers y Davidson la crisis se manifiesta en una sensación ocupacional de "resignación pasiva" o indecisión que lleva a los periodistas a considerar ocupaciones alternativas (2014, p. 1002). Los autores explican que "en el contexto del debate conceptual sobre el profesionalismo periodístico y en el contexto de la crisis actual en la industria del periodismo, nuestros hallazgos enfatizan que, en lugar de ser una profesión emergente, el periodismo en realidad podría ser una profesión en declive" (Meyers \& Davidson, 2014, p. 994). Sin embargo, aún en este contexto de crisis, los periodistas han sido capaces de desarrollar críticas a la situación que sirven para pensar posibles salidas e intervenciones. Para Siapera et al., (2015), "la formulación de estas críticas muestra que el campo se ha movido desde el momento de la crisis hacia la elaboración de nuevas posibilidades para una realidad diferente. Si bien pueden diferir en sus estimaciones y detalles, [estas críticas] están claramente formuladas y son coherentes internamente, mientras que, además, se incorporan directamente a las prácticas periodísticas" (2015, p. 460). 
Para varios investigadores, estas experiencias descritas colaboran en la constante redefinición de los límites de la profesión, como el auge del emprendimiento (Alvear \& Mellado, 2018; Ananny \& Crawford, 2015; Beckers et al., 2017; Conboy \& Eldridge, 2014; Graves \& Konieczna, 2015; Schmitz Weiss, de Macedo Higgins Joyce, Harlow, \& Alves, 2018; Wahl-Jorgensen, 2018). Para Conboy y Eldridge (2014) son evidentes las huellas de un nuevo tipo de periodismo que no descuida sus valores tradicionales y su rol cívico, pero que al mismo tiempo es más reflexivo y se involucra con sus audiencias:

Presente en estos discursos hay una mezcla de periodismo que defiende su necesidad social y cívica en respuesta a la investigación, un periodismo más abierto que se relaciona con una audiencia de ciudadanos y las nuevas oportunidades que ofrecen (...). Estos elementos indican una revitalización de una autopercepción clásica e idealizada del periodismo (p. 8).

Schmitz Weiss y sus colegas (2018) examinaron diferentes emprendimientos de noticias en Latinoamérica y concluyeron que la innovación periodística puede ser conceptualizada "como un producto a través de nuevas formas de narración, así como un proceso a través de colaboraciones con otros y prácticas de participación comunitaria" (p. 96). Sin embargo, todavía queda por responder la pregunta de si estas organizaciones innovadoras serán capaces de sostenerse en el tiempo y, eventualmente, resolver los problemas de la pérdida de los valores del periodismo y sostener una agenda independiente (Schmitz Weiss et al., 2018; Zuluaga Trujillo \& Gómez Montero, 2019).

En suma, la crisis de la industria actúa directamente sobre la definición de la profesión; la noticia, como producto industrial, comienza a regirse por valores propios de un período marcado por la velocidad y circulación. Como respuesta a ello, los periodistas que sobreviven buscan alternativas de salida, dentro o fuera de las organizaciones de noticias, que los lleva en muchos casos a trabajar en condiciones precarias marcadas por imperativos comerciales. Sin embargo, las herramientas digitales y los nuevos formatos también contribuyen a la consolidación de un tipo de periodismo más reflexivo que, ahora, conoce mucho más a quién le está hablando y cómo hacerlo.

\section{Impacto social y prácticas de consumo}

Los estudios también han analizado el impacto social de la crisis de los diarios en las dinámicas institucionales, la propiedad de los medios, la responsabilidad y la confianza pública (Conboy \& Eldridge, 2014; Cornia, 2016; Fang, 2017; Goyanes \& Rodríguez-Castro, 2018; Hassid \& Repnikova, 2016; Mor \& Reich, 2018; Schlesinger \& Doyle, 2015; Starke, Naab, \& Scherer, 2016). Otras investigaciones han examinado la intersección de los procesos institucionales y de consumo al abordar las 
transformaciones en las relaciones entre los periodistas y el público (Carlson \& Usher, 2016; Holton, Coddington, \& Gil de Zúñiga, 2013; Johnson \& Kelling, 2018; Mellado, Márquez-Ramírez, Mick, Oller Alonso, \& Olivera 2017; Nelson, 2018; Wadbring \& Bergström, 2017).

Por un lado, varios estudios coinciden en que los cambios tecnológicos per se no se constituyen como fuente de pluralismo en la industria ni aseguran la autonomía de la prensa (Ananny, 2014; Becerra, 2015; Cornia, 2016; Doyle, 2015; Fang, 2017; Goyanes \& Rodríguez-Castro, 2018; Porto, 2010); por el contrario, en algunos casos aparecen como un obstáculo. Para Cornia (2016), quien evalúa el impacto de la transición digital en la estructura del mercado italiano, si bien "la transición digital conlleva menores barreras tecnológicas para ingresar al campo de las noticias [...] las barreras del mercado y, sobre todo, las barreras políticas aún dificultan la entrada y la consolidación de los recién llegados" (2016, p. 191). Por lo tanto, la autonomía de prensa, según afirma Ananny:

es el resultado de cómo los periodistas navegan por un conjunto emergente de fuerzas externas e internas, por ejemplo, al permitir o no a los lectores interactuar con el contenido de las noticias y cómo se encuentran con su público en las redes sociales. Sin embargo, parece que, al menos en las políticas oficiales que regulan el uso de las redes sociales por parte de los trabajadores de las noticias, las organizaciones de noticias están adoptando relaciones utilitarias, defensivas y fundamentalmente conservadoras para el público, que continúan buscando la libertad de los lectores (2014, p. 948).

Por el otro lado, las investigaciones destacan los posibles beneficios que el vínculo entre la tecnología y las instituciones de medios pueden generar. La tecnología juega un rol transversal en la industria (García-Avilés, CarvajalPrieto, Arias-Robles, \& De Lara-González, 2019). Para Hassid y Repnikova el vínculo que los periodistas del diario en papel e Internet establecen en China es "simbiótico", dado que "a diferencia de los países occidentales, donde los medios generalmente administran su propia supervivencia financiera, en China, todos los medios tradicionales siguen siendo propiedad del Estado" (2016, p. 892). En esa misma línea, Nelson (2018) propone pensar un vínculo más participativo entre los medios, Internet y las audiencias; algo que favorece el control de las actividades estatales o que puede lograr un impacto positivo en la reducción y el control de la corrupción (Starke et al., 2016). El estudio de Starke y sus colegas "apoya las esperanzas de que los medios de comunicación libres cumplan con sus tareas de responsabilizar a los funcionarios públicos, crear una sociedad más transparente, disuadir a los actores corruptos de acciones ilegales al aumentar el riesgo de detección y reforzar las leyes anticorrupción" (2016, p. 4713). Al mismo 
tiempo, estos vínculos también impactan en la estructura interna de las empresas de noticias dándole mayor agilidad y una actitud experimental en la cultura organizacional (Carlson \& Usher, 2016).

En lo que refiere al rol que la tecnología juega en relación a la confianza en los medios (Chadha \& Koliska, 2015; Fang, 2017; Holton et al., 2013), un estudio de Mor y Reich (2018) propone analizar el caso de DocumentCloud, una plataforma que alienta a los periodistas a publicar documentos e información que respalden sus historias. Para los autores, la plataforma otorga transparencia al periodismo dando a conocer la evidencia, ampliando la información e invitando a la audiencia a participar de la verificación de la información, algo que "implementado en una escala mayor puede ayudar a reconstruir la confianza pública en los medios de noticias" (Mor \& Reich, 2018, p. 1103). Sin embargo, Fang (2017) afirma que, en determinados contextos, las tecnologías digitales también pueden mostrar su "lado oscuro" al desempeñar un papel importante en la promoción de la desconfianza y los ataques a los periodistas permitiendo su "arrastre y el hostigamiento (...) y promoviendo la visibilidad y popularidad de los atacantes más radicales en la comunidad" (2017, p. 59).

Esta tensión también se ve reflejada el surgimiento de canales alternativos y nuevas narrativas (Robledo-Dioses \& Atarama-Rojas, 2018; Starbird, 2017) y el rol de las redes sociales en su distribución (Budak, 2019; Johnson \& Kelling, 2018; Starbird, 2017). Esta última autora describe cómo circulan y se relacionan las noticias desde estos medios alternativos con la propagación de noticias falsas y las teorías conspirativas y pseudociencias que, en última instancia, funcionan como promotores de agendas políticas subyacentes. "La estructura y la dinámica del ecosistema de medios alternativos proporcionan evidencia de [las] tácticas de desinformación intencionales diseñadas no para difundir una ideología específica sino para socavar la confianza en la información en general" (Starbird, 2017, p. 239). Esto coincide con lo que propone Budak (2019), quien explica que durante las elecciones presidenciales de 2016 en Estados Unidos los individuos reportaron haber visto, leído o escuchado información que se alinea más con la propuesta de agenda de los medios de noticias falsas que con la de los medios tradicionales. Para la autora, eso se explica en parte por una "similitud en los temas populares entre los sitios de noticias falsas y los tradicionales" pero que cuando se analiza el tratamiento de los temas políticos, los medios tradicionales lo hicieron débilmente, enfocados en cubrir los escándalos de los candidatos (Budak, 2019, p. 148). Sin embargo, la emergencia de canales alternativos también puede traer consigo la exploración de nuevas narrativas transmedia que podrían resultar una oportunidad para la reinvención del periodismo en la 
región (Fernández Castrillo, 2014; Renó, 2011; Albarello, 2013). Como proponen Robledo-Dioses y Ataram Rojas:

el periodismo se enfrenta a diversas audiencias con características distintas muy marcadas entre sí, lo que implica que el proceso generalista tradicional de producción y difusión de información está ya desfasado y debe ser reemplazado por un proceso narrativo que tenga como centro al usuario y que, a su vez, pensando en este, no descuide la calidad de los contenidos (2018, p. 124).

Como resultado de lo expuesto, se puede comprender la crisis de la industria desde los cambios que ocurren en las dinámicas sociales de inserción de las organizaciones de noticias. La tecnología, lejos de convertirse en un factor que pueda otorgar sin discusión pluralidad a la información, juega un rol fundamental en la definición del vínculo entre la prensa y los ciudadanos. En la constante redefinición de los valores tradicionales de la prensa y la confianza en los medios de noticia tradicionales, surgen nuevas narrativas que cuestionan cómo se produce y circula la información en estos tiempos.

Finalmente, aunque en menor medida, algunos especialistas se han enfocado en comprender qué cambios ocurren en el consumo de la información y cómo ello se ve afectado por la crisis del diario en papel: quiénes consumen noticias, cómo las consumen y dónde lo están haciendo son las principales preguntas que articulan los estudios (Albarello 2020; Bipat, Wilson, Kurniawan, Choi, \& Starbird,2019; Chyi \& Tenenboim, 2019; Nelson, 2018; Papathanassopoulos et al., 2013; Pellegrini, Bradley, Swift, \& Boals, 2015; Thurman, 2018; Thurman \& Fletcher, 2019; Wadbring \& Bergström, 2017).

La discusión se centró principalmente alrededor de la caída de la lectura del diario, especialmente entre los jóvenes. Thurman y Fletcher (2019) analizan el caso británico mediante la evolución del tiempo destinado (time spent) a once plataformas de noticias en la que se evidencia una notoria caída de $40 \%$ en el tiempo dedicado a los diarios en los últimos años. "Esta reducción se produjo a pesar de que la distribución online redujo los costos de consumo de contenido, tanto en términos financieros - al proporcionar acceso gratuito en muchos casos - como con respecto a la comodidad y la velocidad" (2019, p. 14). Según Wadbring \& Bergström (2017), la caída de lectores de diarios impresos es independiente de si incluimos o no las ediciones web y la explicación parece ser generacional. Para los autores "las generaciones más jóvenes tienden a abandonar los periódicos impresos sin importar su costo" (2017, p. 185). Sin embargo, todas las generaciones han incrementado su consumo de noticias en televisión, aunque solo de manera modesta entre las generaciones más jóvenes, demostrada por la estabilidad de la audiencia en el tiempo (Wadbring \& Bergström, 2017). En 
este contexto, Papathanassopoulos y sus colegas concluyen que incluso aunque Internet haya cambiado toda la comunicación dando a las personas más fuentes de información, la televisión sigue siendo la opción de noticias más popular. Esto sugiere que las personas eligen entre la TV, los diarios o la web para contar con "contenido paralelo de noticias" (Papathanassopoulos et al., 2013, p. 701). En América Latina esto también puede explicarse en parte por el aumento de la circulación de diarios impresos gratuitos (Bakker, 2012). García Rubio (2013) comenta que, en México, "los diarios con mayor circulación son los populares, gratuitos y algunos de información general. Es posible inferir que el lectorado mexicano ha optado por una prensa económica o bien gratuita, la cual está asociada a bajos estándares de calidad" (p. 90).

\section{ENFOQUES TEÓRICOS Y METODOLÓGICOS}

Las investigaciones sobre la crisis de la prensa han sido heterogéneas en cuanto a sus enfoques conceptuales y al conjunto de técnicas utilizadas. A continuación, se describirán brevemente aquellos aspectos sobresalientes. Los investigadores han encuadrado sus análisis particularmente en la comprensión de los modelos de negocio de los medios (Brandstetter \& Schmalhofer, 2014; Doyle, 2013; Ihlström Eriksson et al., 2016; Kolo, 2016; Picard, 2014; van der Burg \& Van den Bulck, 2017) para comprender, en muchos casos, la transición digital que muchas de estas instituciones de noticias están experimentando (Rothmann \& Koch, 2014; Villi \& Hayashi, 2017). Otros investigadores han conceptualizado el fenómeno desde el giro sociológico de la economía de los medios (Schlesinger \& Doyle, 2015). En ese sentido, la sociología ha sido predominante con diferentes texturas: desde la óptica de la sociología del trabajo (Ekdale, Tully, Harmsen, \& Singer, 2015) hasta la sociología institucional y el comportamiento organizacional (Ananny, 2014; Ananny \& Crawford, 2015; Davidson \& Meyers, 2016; Graves \& Konieczna, 2015). Una mirada innovadora es la de Usher (2015), quien sitúa su investigación desde la teoría social y cultural del espacio para analizar cómo cambian las dinámicas laborales en la redacción de un diario en Miami. Quienes han abordado el posible impacto de la crisis de la prensa escrita en la esfera pública y la democracia han preferido enfoques conceptuales provenientes de la economía política de los medios y la comunicación política (Cornia, 2016; Retegui, 2013; Starke et al., 2016). También desde los estudios sobre periodismo se han conceptualizado los nuevos horizontes de la prensa, sus valores y dinámicas (Ananny, 2014; Bachmann \& Harlow, 2012; Hassid \& Repnikova, 2016; Mor \& Reich, 2018).

En cuanto a las técnicas y métodos utilizados, se han recurrido mayormente a los estudios de casos para comprender los objetos en cuestión. Por ejemplo, para 
describir cómo la crisis afecta las percepciones de los periodistas, las dinámicas laborales y los vínculos con las audiencias, las investigaciones han recurrido a las historias de vida de los reporteros (Davidson \& Meyers, 2016), a las entrevistas en profundidad (Ananny \& Crawford, 2015; Bardan, 2015; De La Torre \& Dillon, 2012; Gómez \& Louzada, 2017; Sarrimo, 2017; Villi \& Hayashi, 2017), en menor medida a encuestas (O'Donnell et al., 2016), a etnografías (Boczkowski et al., 2019; Graves \& Konieczna, 2015; Usher, 2015), y a grupos focales (Schmitz Weiss et al., 2018). Retegui (2020) combinó un acercamiento etnográfico al interior de la redacción del diario La Nación en Argentina con entrevistas en profundidad y análisis bibliográfico para conocer el impacto de los indicadores de medición de las noticias digitales en la labor periodística.

Aunque es una técnica utilizada con menor frecuencia, los enfoques textuales y de contenido también han servido como herramientas metodológicas fructíferas en el campo (Alvear \& Mellado, 2018; Ananny, 2014; Bachmann \& Harlow, 2012; Brandstetter \& Schmalhofer, 2014; Carlson \& Usher, 2016; Conboy \& Eldridge, 2014; Mor \& Reich, 2018; Starbird, 2017). Por ejemplo, Bachmann y Harlow (2012) realizaron un análisis de contenido de diecinueve diarios en Latinoamérica para comprender cómo estos medios están respondiendo a las nuevas exigencias multimedia. Algunos años después, Barredo-Ibáñez y DíazCerveró (2017) también realizaron análisis de contenido para evaluar, en base a una serie de indicadores de calidad, la interactividad en cibermedios colombianos, mexicanos y ecuatorianos.

Finalmente, se ha recurrido en menor medida a estudios longitudinales y transnacionales (Schmitz Weiss et al., 2018; Starke et al., 2016; van der Burg \& Van den Bulck, 2017). Es destacable el trabajo realizado por Schmitz Weiss y sus colegas (2018) quienes, para comprender más acerca de las organizaciones de noticias emprendedoras en la región, realizaron grupos focales con participantes de Argentina, Brasil, Chile, El Salvador, México, Nicaragua, Perú, y Venezuela. Otros estudios, mediante el uso y análisis datos cuantitativos secundarios, proponen una perspectiva que permite la comparación y la delimitación del fenómeno en un sentido más amplio. Por ejemplo, en su estudio sobre el impacto de la libertad de expresión, el acceso a Internet y la prestación de servicios gubernamentales en línea en la corrupción, Starke, Naab y Scherer (2016) analizan y comparan los resultados en 157 países durante los años 2003, 2008 y 2013. 


\section{HACIA UNA COMPRENSIÓN LATINOAMERICANA DE LA CRISIS DE LA PRENSA ESCRITA}

Hasta aquí hemos revisado la literatura reciente sobre la crisis del diario en papel, sus principales declinaciones temáticas y enfoques metodológicos. En este apartado describiremos una posible agenda que contemple las fortalezas de las investigaciones examinadas, pero también cómo se pueden sortear las posibles limitaciones considerando la particular perspectiva y experiencia latinoamericana.

Es valioso destacar que, en la mayoría de las investigaciones, la pregunta por el futuro del periodismo y de la prensa escrita ha sido circunscrita a una temporalidad acotada que gira en torno a casos específicos. En otras palabras, los objetos de investigación se han corrido del qué pasaría si... - una preocupación bastante extendida desde la aparición de Internet- para centrarse en lo que efectivamente ha ocurrido o está ocurriendo en un territorio determinado. Las investigaciones relevadas están basadas principalmente en casos con preguntas concretas, que evalúan hechos puntuales (mudanzas, despidos, cambios en las estrategias de negocio, caídas en la circulación, cierre de medios, entre otros) y en menor medida son predictivos del futuro. La crisis ya no es una amenaza; por el contrario, se convierte en parte de la cotidianeidad de los medios.

No obstante, la pregunta específica y los análisis de casos dejan abierta la oportunidad para obtener conocimiento sistemático que permita realizar comparaciones y análisis multidimensionales más complejos. Desde esa perspectiva, una forma de darle sentido a los hallazgos individuales debería considerar la inclusión de una mirada histórica que atienda a los cambios de las percepciones y discursos con parámetros comparables en el tiempo y en diferentes territorios. Como vimos hasta aquí, la enorme mayoría de los estudios recaba datos de una única fuente de información. Cuando las investigaciones cuentan con dos fuentes o más, generalmente están usadas de manera complementaria a la primera. Para el caso latinoamericano, esto se puede deber en parte a la dificultad de contar con datos sistemáticos y confiables, como explica García Rubio para México en donde:

Hasta ahora no se ha logrado crear un índice nacional y estatal exhaustivo de publicaciones, así como tampoco contar con cifras claras sobre la circulación de la prensa. Respecto de esto último, varios investigadores han subrayado el oscurantismo en tirajes, poniendo en duda la confiabilidad de las cifras de circulación y tirajes de los impresos (2013, p. 69).

Desde el punto de vista de las dimensiones de análisis, mucho se ha escrito sobre las dimensiones productivas de la prensa y menos se ha indagado sobre la recepción, con valiosas excepciones (Focás et al., 2016; Boczkowski et al., 2019; Mastrini et al., 2014; Albarello 2020). Es necesario preguntarse qué ocurre en el consumo de la información y qué rol tienen los soportes (sean impresos o digitales) 
en el contexto más amplio de consumo de medios de comunicación y, desde luego, en la vida cotidiana de las personas. Podríamos afirmar entonces que el término crisis es un concepto o un estado de situación que solo le ha interesado analizar a la industria y a la academia. Si bien son crecientes las investigaciones que indagan el consumo de noticias en plataformas digitales (Mitchelstein \& Boczkowski, 2009), la falta de investigaciones que se centren en comprender el consumo de información -y noticias en papel, en particular - desde el lente de las prácticas individuales y colectivas sugiere la hipótesis de que, probablemente, para la voz de las audiencias se trate menos de una crisis y más de nuevas experiencias (o mutaciones de algunas preexistentes) en el consumo de información.

La literatura sobre la crisis de la prensa escrita ha sido constantemente innovadora en su incorporación de la información proveniente sobre los negocios y la publicidad, especialmente para abordar cómo funcionan los nuevos negocios con base digital, sumando complejos modelos econométricos para comprender la rentabilidad de las empresas de medios, pero los estudios sobre el consumo del diario en papel permanecen anclados a lo que Couldry (2012) llama mediacentrismo. En otras palabras, estas investigaciones se han enfocado en las dimensiones que se desprenden de los medios, en tanto dispositivos tecnológicos, olvidando en muchos casos lo que ocurre en la vida cotidiana de las personas. A propósito de ello, algunos trabajos recientes están reorientando los enfoques conceptuales y teóricos para comprender qué sucede en la intersección material entre el dispositivo tecnológico, la información y las prácticas cotidianas (Boczkowski et al., 2019; Fortunati, Taipale, \& Farinosi, 2015; Kormelink \& Meijer, 2019). Más allá de explicar la crisis, lo que estas investigaciones proponen son nuevas hipótesis que exploran las razones por las que las personas continúan consumiendo el diario en papel, los motivos y contextos que subyacen a prácticas altamente habitualizadas.

Finalmente, la evidencia sugiere que es necesaria una perspectiva comparada para abordar la crisis. Las investigaciones aquí descritas se centran mayormente en el hemisferio norte, específicamente en los Estados Unidos. Sin embargo, a partir de algunas investigaciones relevadas en este estudio (Bachmann \& Harlow, 2012; Barrios, 2010; Schmitz Weiss et al., 2018) queda de manifiesto la factibilidad de construir escenarios comparativos en América Latina, en parte por la colaboración académica de investigadores/as de la región.

A sabiendas de ello, aun cuando todavía resulta sumamente complejo definir los indicadores empíricos para evaluar los cambios e impactos de la prensa en la democracia (Schudson, 2010), es importante continuar explorando el vínculo que tiene, o puede tener, la prensa, el periodismo y los medios de 
comunicación en la formación de una responsabilidad cívica y un fortalecimiento de los valores democráticos (Porto \& Hallin, 2009; Rojas, 2006) en contextos en donde la concentración de la propiedad de los medios domina la escena (Becerra \& Mastrini, 2017).

\section{CONCLUSIÓN}

Comenzamos este ensayo con dos objetivos. El primero fue discutir cómo la academia, desde la publicación de Siles y Boczkowski (2012), ha abordado la crisis del diario impreso en la última década. A partir de ello, dimos cuenta de sus enfoques temáticos, a saber, económicos, laborales y sociales, y describimos sus perspectivas y limitaciones teóricas y metodológicas. El segundo objetivo, que surge de la sistematización de la literatura sobre el tema, fue dilucidar oportunidades y ofrecer un marco y agenda posible para abordar la problemática desde Latinoamérica, considerando la escasa, aunque muy relevante, participación en la discusión.

Las investigaciones analizadas aquí manifiestan dos hechos fundamentales. En primer lugar, evidencian una divergencia en la aproximación al objeto: la crisis del diario y el consumo de información están fuertemente caracterizados por los investigadores en su dimensión informativa y organizadas en relación con los medios como dispositivos tecnológicos. Del otro lado del puente quedan las prácticas de consumo y apropiación de esos medios por parte de las audiencias en su vida cotidiana. Esta divergencia entre los medios y las audiencias podría iluminar nuevas preguntas de investigación que giren en torno a su imbricación en la vida social como, por ejemplo: ¿Qué vínculos emergen entre los modos de acceso a las noticias (papel, digital, televisión, radio, otros) y las prácticas de consumo en la vida cotidiana? A partir de ello: ¿qué rituales se circunscriben y dan forma a la experiencia de consumo de noticias en la región? Además: ¿cómo las audiencias perciben los cambios de estrategia y las transiciones de los medios de noticias? ¿Qué dinámicas sociales más amplias permiten caracterizar los modos de consumimos noticias en la actualidad?

En segundo lugar, el tópico de la crisis de la prensa ha sido profusamente abordado por investigadores en el hemisferio norte, especialmente en los Estados Unidos y Europa. Continuando con la metáfora del puente, es necesario construir uno que conecte las experiencias de la crisis del diario en otros territorios con aquellos hallazgos y particularidades de países desarrollados. América Latina es una región en la que los diarios impresos tienen una tradición centenaria: jugaron un rol fundamental desde la independencia (Piccato, 2010) hasta la actualidad. Sin embargo, la heterogeneidad de la región, como la relativamente reciente democratización y la inestabilidad económica, pueden resignificar 
tanto el contexto como las consecuencias de la crisis de la prensa. A su vez, examinar las diferencias entre la decadencia del diario en papel en democracias industrializadas y en países en desarrollo llevará a una comprensión más acabada de esta transición en su totalidad.

Finalmente, se espera que este ensayo funcione como articulador de lo que hasta ahora han sido dos mundos. Particularmente, que sirva como impulsor de nuevas investigaciones con base en lo que ya se conoce en un contexto en el que la investigación en el campo de la comunicación está experimentando una época de globalización tanto institucional como disciplinar (Waisbord, 2019), pero que también se desafíen, impugnen y reescriban los marcos metodológicos y conceptuales a fin de dar cuenta de las peculiaridades de la prensa en Latinoamérica.

\section{REFERENCIAS}

Albarello, F. (2013). Carlos Scolari. Narrativas transmedia. Cuando todos los medios cuentan: Barcelona, Deusto, 2013, 342 pp. ISBN 978-84-234-1336-2 (Carlos Scolari. Transmedia narratives. When all the media count). Austral Comunicación, 2(2), 247-249. https://doi.org/10.26422/aucom.2013.0202.alba

Albarello, F. (2020). De la hegemonía al nicho: desplazamiento de medios tradicionales en rutinas informativas de un grupo de universitarios argentinos (From hegemony to the niche: displacement of traditional media in informative routines of a group of Argentine university students). Dixit, (32), 46-60. https://doi.org/10.22235/d.vi32.1946

Alvear, L. \& Mellado, C. (2018). La presencia de rol cívico, de servicio y de infoentretenimiento en las noticias de cultura y espectáculos del periodismo chileno (The Presence of Civic, Service and Infotainment Roles in the News about Culture and Show Business of Chilean Journalism). Palabra Clave, 21(2), 338-363. https://doi.org/10.5294/pacla.2018.21.2.4

Ananny, M. (2014). Networked Press Freedom and Social Media: Tracing Historical and Contemporary Forces in Press-Public Relations. Journal of Computer-Mediated Communication, 19(4), 938-956. https://doi.org/10.1111/jcc4.12076

Ananny, M. \& Crawford, K. (2015). A Liminal Press: Situating news app designers within a field of networked news production. Digital Journalism, 3(2), 192-208. https://doi.org/10.1080/21670811.2014.922322

Anderson, B. (2006). Imagined communities: Reflections on the origin and spread of nationalism. London: Verso books.

Angelucci, C. \& Cagé, J. (2016). Newspapers in times of low advertising revenues. Centre for Economic Policy Research, DP11414. Retrieved from https://portal.cepr.org/discussion-paper/357

Bachmann, I. \& Harlow, S. (2012). Interactivity and multimedia in Latin American newspapers: Inroads in an incomplete transition. Cuadernos.info, (30), 41-52. Retrieved from https://cuadernos.info/index.php/CDI/article/view/421/pdf 
Bakker, P. (2012). The rise offree daily newspapers in Latin America. Revistadecomunicación, (11), 129-149. Retrieved from https://revistadecomunicacion.com/es/articulos/2012/Art129-149.html

Bardan, A. (2015). The Dual Model of the Digital Photojournalist: A Case Study on Romanian Photojournalism beyond the Economic Crisis. Journal of Media Research - Revista de Studii Media, 8(21), 19-40. Retrieved from https://www.ceeol.com/search/article-detail?id=6792

Barredo-Ibáñez, D., Díaz-Cerveró, E., Garzón, K. T. P., del Rosario Gómez, R., Pérez, S., Nava, F. E., ... \& Estrada, L. G. (2017). La interactividad en el periodismo digital latinoamericano. Un análisis de los principales cibermedios de Colombia, México y Ecuador (2016) (Interactivity in Latin American digital journalism. An analysis of the main cybermedia of Colombia, Mexico and Ecuador (2016)). Revista Latina de Comunicación Social, (72), 273-294. https://doi.org/10.4185/RLCS-2017-1165

Barrios, M. M. (2010). Los diarios digitales en América Latina: entre la revolución, la redefinición y la frustración (Digital newspapers in Latin America: between revolution, redefinition and frustration). Investigación \& Desarrollo, 15(2). http://rcientificas.uninorte.edu.co/index.php/investigacion/article/viewFile/887/531

Becerra, M. (2015). De la concentración a la convergencia. Políticas de medios en Argentina y América Latina (From concentration to convergence. Media policies in Argentina and Latin America). Buenos Aires: Paidós.

Becerra, M. \& Mastrini, G. (2017). Concentración y convergencia de medios en América Latina (Media concentration and convergence in Latin America). Communiquer. Revue de communication sociale et publique, (20), 104-120. https://doi.org/10.4000/communiquer.2277

Beckers, K., Masini, A., Sevenans, J., van der Burg, M., De Smedt, J., Van den Bulck, H., \& Walgrave, S. (2017). Are newspapers' news stories becoming more alike? Media content diversity in Belgium, 1983-2013. Journalism, 20(12), 1665-1683. https://doi.org/10.1177/1464884917706860

Bipat, T., Wilson, T., Kurniawan, O., Choi, Y. J. S., \& Starbird, K. (2019). It is Not All Fun and Games: Breaking News Consumption on Snapchat. Proceedings of the 52nd Hawaii International Conference on System Sciences. Retrieved from http://hdl.handle.net/10125/59649

Boczkowski, P. J., Mitchelstein, E., \& Suenzo, F. (2019). The Smells, Sights, and Pleasures of Ink on Paper: The Consumption of Print Newspapers During a Period Marked by Their Crisis. Journalism Studies, 21(5), 565-581. https://doi.org/10.1080/1461670X.2019.1670092

Brandstetter, B. \& Schmalhofer, J. (2014). Paid Content: A successful revenue model for publishing houses in Germany? Journalism Practice, 8(5), 499-507. https://doi.org/10.1080/17512786.2014.895519

Budak, C. (2019). What Happened? The Spread of Fake News Publisher Content During the 2016 U.S. Presidential Election. The World Wide Web Conference, 139-150. https://doi.org/10.1145/3308558.3313721

Carlson, M. \& Usher, N. (2016). News Startups as Agents of Innovation: For-profit digital news startup manifestos as metajournalistic discourse. Digital Journalism, 4(5), 563-581. https://doi.org/10.1080/21670811.2015.1076344

Chadha, K. \& Koliska, M. (2015). Newsrooms and Transparency in the Digital Age. Journalism Practice, 9(2), 215-229. https://doi.org/10.1080/17512786.2014.924737 
Chyi, H. I. \& Tenenboim, O. (2019). Charging More and Wondering Why Readership Declined? A Longitudinal Study of U.S. Newspapers' Price Hikes, 2008-2016. Journalism Studies, 20(14), 2113-2129. https://doi.org/10.1080/1461670X.2019.1568903

Cohen, N. S. (2015). Entrepreneurial Journalism and the Precarious State of Media Work. The South Atlantic quarterly, 114(3), 513-533. https://doi.org/10.1215/00382876-3130723

Conboy, M. \& Eldridge, S. A. (2014). Morbid symptoms. Between a dying and a re-birth (apologies to Gramsci).Journalism Studies, 15(5), 566-575. https://doi.org/10.1080/1461670X.2014.894375

Cornia, A. (2016). TV-centrism and politicisation in Italy: obstacles to new media development and pluralism. Media Culture \& Society, 38(2), 175-195. https://doi.org/10.1177/0163443715594035

Couldry, N. (2012). Media, Society, World: Social Theory and Digital Media Practice. Polity.

Davidson, R. \& Meyers, O. (2016). “Should I Stay or Should I Go?”: Exit, voice and loyalty among journalists.Journalism Studies, 17(5), 590-607. https://doi.org/10.1080/1461670X.2014.988996

De La Torre, L. \& Dillon, A. (2012). Comunicación, redes sociales y democracia en la mirada de periodistas argentinos (Communication, Social Networks and Democracy According to Journalists in Argentina). Cuadernos. info, (30), 61-72. https://doi.org/10.7764/cdi.30.423

Doyle, G. (2013). Re-Invention and Survival: Newspapers in the Era of Digital Multiplatform Delivery. Journal of Media Business Studies, 10(4), 1-20. https://doi.org/10.1080/16522354.2013.11073569

Doyle, G. (2015). Why Ownership Pluralism Still Matters in a Multi-platform World. In P. Valcke, M. Sükösd, \& R. G. Picard (Eds.), Media Pluralism and Diversity: Concepts, Risks and Global Trends (pp. 297-309). Palgrave Macmillan UK. https://doi.org/10.1057/9781137304308_18

Ekdale, B., Tully, M., Harmsen, S., \& Singer, J. B. (2015). Newswork Within a Culture of Job Insecurity: Producing news amidst organizational and industry uncertainty. Journalism Practice, 9(3), 383-398. https://doi.org/10.1080/17512786.2014.963376

Evans, S. K. (2016). Staying Ahead of the Digital Tsunami: The Contributions of an Organizational Communication Approach to Journalism in the Information Age. The Journal of communication, 66(2), 280-298. https://doi.org/10.1111/jcom.12217

Fang, K. (2017). "Guard against fire, theft, and journalists": the public against the press in China. Media Asia, 44(1), 1-6. https://doi.org/10.1080/01296612.2017.1374628

Fernández Castrillo, C. (2014). Prácticas transmedia en la era del prosumidor: Hacia una definición del Contenido Generado por el Usuario (CGU) (Transmedia practices in the prosumer era: Towards a definition of User Generated Content (UGC)). CIC. Cuadernos de información y comunicación, 19, 53-67. https://doi.org/10.5209/rev_CIYC.2014.v19.43903

Focás, B., Alarcón, C., Polesel, C. R., Galar, S., Del Palacio Montiel, C., Browne Mönckeberg, M., ... \& Varjão, S. (2016). (In) seguridad, medios y miedos: una mirada desde las experiencias $y$ las prácticas cotidianas en América Latina ((In) security, media and fears: a look from daily experiences and practices in Latin America). Cali, Colombia: Universidad Icesi.

Fortunati, L., Taipale, S., \& Farinosi, M. (2015). Print and online newspapers as material artefacts. Journalism, 16(6), 830-846. https://doi.org/10.1177/1464884914545439

García-Avilés, J. A., Carvajal-Prieto, M., Arias-Robles, F., \& De Lara-González, A. (2019). Journalists' views on innovating in the newsroom: Proposing a model of the diffusion of innovations in media outlets. The Journal of Media Innovations, 5(1), 1-16.

https://doi.org/10.5617/jomi.v5i1.3968 
García Rubio, C. I. (2013). Radiografía de la prensa diaria en México en 2010 (The daily press in Mexico in 2010). Comunicación y sociedad, (20), 65-93. https://doi.org/10.32870/cys.v0i20.217

Godoy, J. A. R. \& Ospina, A. M. P. (2014). El sector de medios impresos en Colombia (The print media industry in Colombia: an interpretation of its financial situation). Entramado, 10(1), 30-54. https://revistas.unilibre.edu.co/index.php/entramado/article/view/3480

Gómez, E. F. R. \& Louzada, C. F. (2017). ¿Es rentable el periodismo de calidad? Disposición al pago en Brasil y España (Is quality journalism profitable? Intent to pay in Brazil and Spain). Transinformação, 29(2), 211-220. https://doi.org/10.1590/2318-08892017000200008

Gómez-Luna, E., Fernando-Navas, D., Aponte-Mayor, G., \& Betancourt-Buitrago, L. A. (2014). Metodología para la revisión bibliográfica y la gestión de información de temas científicos, a través de su estructuración y sistematización (Literature review methodology for scientific and information management, through its structuring and systematization). Dyna, 81(184), 158-163. Retrieved from http://dyna.medellin.unal.edu. co/en/verResumenEN.php?id_articulo=v81n184a21

Goyanes, M. \& Rodríguez-Castro, M. (2018). Commercial Pressures in Spanish Newsrooms: Between love, struggle and resistance. Journalism Studies, 20(8), 1088-1109. https://doi.org/10.1080/1461670X.2018.1487801

Graves, L. \& Konieczna, M. (2015). Qualitative Political Communication| Sharing the News: Journalistic Collaboration as Field Repair. International Journal of Communication Systems, 9, 19. Retrieved from http://ijoc.org/index.php/ijoc/article/view/3381

Habermas, J. (2015). The Theory of Communicative Action: Lifeworld and Systems, a Critique of Functionalist Reason. John Wiley \& Sons.

Hassid, J. \& Repnikova, M. (2016). Why Chinese print journalists embrace the Internet. Journalism, 17(7), 882-898. https://doi.org/10.1177/1464884915592405

Hofstetter, B. \& Schoenhagen, P. (2017). When Creative Potentials are Being Undermined By Commercial Imperatives: Change and resistance in six cases of newsroom reorganisation. Digital Journalism, 5(1), 44-60. https://doi.org/10.1080/21670811.2016.1155966

Holton, A. E., Coddington, M., \& Gil de Zúñiga, H. (2013). Whose News? Whose Values?: Citizen journalism and journalistic values through the lens of content creators and consumers. Journalism Practice, 7(6), 720-737. https://doi.org/10.1080/17512786.2013.766062

Ihlström Eriksson, C., Akesson, M., \& Lund, J. (2016). Designing Ubiquitous Media Services: Exploring the Two-Sided Market of Newspapers. Journal of theoretical and applied electronic commerce research, 11(3), 1-19. https://doi.org/10.4067/S0718-18762016000300002

Johnson, B. G. \& Kelling, K. (2018). Placing Facebook: “Trending," “Napalm Girl,” “fake news" and journalistic boundary work. Journalism Practice, 12(7), 817-833. https://doi.org/10.1080/17512786.2017.1349546

Knight Center LatAm Journalism Review. (2018, mayo 6). Crisis de periódicos llega al Diario de Pernambuco, el de más antigua circulación en América Latina. Knight Center Journalism Review. https://latamjournalismreview.org/es/articles/crisis-de-periodicos-llega-aldiario-de-pernambuco-el-de-mas-antigua-circulacion-en-america-latina/ 
Kolo, C. (2016). A Long-term View on the Business Model of Newspaper Publishing: International Comparison and Quantitative Modelling for Germany as Case in point. Westminster Papers in Communication and Culture, 11(1). https://doi.org/10.16997/wpcc.217

Kormelink, T. G. \& Meijer, I. C. (2019). Material and sensory dimensions of everyday news use. Media, Culture \& Society, 41(5), 637-653. https://doi.org/10.1177/0163443718810910

Mastrini, G., Marino, S., Becerra, M., Gadano, J., Bieda, T., Bizberge, A., Perea, R., \& Rodríguez Miranda, C. (2014). Uso y consumo de la Televisión Digital Terreste en Argentina. Un estudio en los municipios de San Fernando y Quilmes (Use and consumption of Digital Terrestrial Television in Argentina. A study in the municipalities of San Fernando and Quilmes). Quilmes, Argentina: Universidad Nacional de Quilmes.

Mellado, C., Márquez-Ramírez, M., Mick, J., Oller Alonso, M., \& Olivera, D. (2017). Journalistic performance in Latin America: A comparative study of professional roles in news content. Journalism, 18(9), 1087-1106. https://doi.org/10.1177/1464884916657509

Meyers, O. \& Davidson, R. (2014). The journalistic structure of feeling: An exploration of career life histories of Israeli journalists. Journalism, 15(8), 987-1005. https://doi.org/10.1177/1464884913512930

Meyers, O. \& Davidson, R. (2016). Conceptualizing Journalistic Careers: Between Interpretive Community and Tribes of Professionalism. Sociology Compass, 10 (6), 419-431. https://doi.org/10.1111/soc4.12376

Mitchelstein, E. \& Boczkowski, P. J. (2009). Between tradition and change: A review of recent research on online news production. Journalism, 10(5), 562-586. https://doi.org/10.1177/1464884909106533

Mor, N. \& Reich, Z. (2018). From “Trust Me" to "Show Me” Journalism: Can DocumentCloud help to restore the deteriorating credibility of news? Journalism Practice, 12(9), 1091-1108. https://doi.org/10.1080/17512786.2017.1376593

Nelson, J. L. (2018). And Deliver Us to Segmentation: The growing appeal of the niche news audience. Journalism Practice, 12(2), 204-219. https://doi.org/10.1080/17512786.2017.1378588

O’Donnell, P., Zion, L., \& Sherwood, M. (2016). Where do journalists go after newsroom job cuts? Journalism Practice, 10(1), 35-51. https://doi.org/10.1080/17512786.2015.1017400

Papathanassopoulos, S., Coen, S., Curran, J., Aalberg, T., Rowe, D., Jones, P., Rojas, H., \& Tiffen, R. (2013). Online Threat, But Television is Still Dominant: A comparative study of 11 nations' news consumption. Journalism Practice, 7(6), 690-704. https://doi.org/10.1080/17512786.2012.761324

Pellegrini, P., Bradley, D., Swift, K., \& Boals, T. (2015). Cross Platform Media Measurement: Mobile and Desktop Online Measurement Comparisons. Paper presented at the Print and Digital Research Forum 2015, London. Retrieved from https://www.pdrf.net/wpcontent/uploads/2015/10/10_Syn43Pellegrini.pdf

Picard, R. G. (2014). Twilight or New Dawn of Journalism?: Evidence from the changing news ecosystem. Journalism Practice, 8(5), 488-498. https://doi.org/10.1080/17512786.2014.905338

Piccato, P. (2010). Public sphere in Latin America: a map of the historiography. Social History, 35(2), 165-192. https://doi.org/10.1080/03071021003795055 
Porto, M. P. (2010). The changing landscape of Brazil's news media. In D.A. Levy \& R. Kleis Nielsen (Eds.), The changing business of journalism and its implications for democracy (pp. 107-124). Oxford, UK: Reuters Institute. Retrieved from https://reutersinstitute. politics.ox.ac.uk/sites/default/files/2017-11/The\%20Changing\%20Business\%20of\%20 Journalism\%20and\%20its\%20Implications\%20for\%20Democracy.pdf\#page=113

Porto, M. P. \& Hallin, D. C. (2009). Media and Democratization in Latin America. The International Journal of Press/Politics, 14(3), 291-295. https://doi.org/10.1177/1940161209336231

Reich, Z. (2018). The decline in orally negotiated news: Revisiting (again) the role of technology in reporting. New Media \& Society, 20(11), 4116-4134. https://doi.org/10.1177/1461444818768064

Renó, D. P. (2011). Periodismo, redes sociales y transmediación (Journalism, social networks and transmedia). Razón y palabra, 16(78).

Retegui, L. M. (2013). La caída constante en las ventas del diario Clarín (1995-2012). Un análisis desde la economía política de la comunicación (The constant drop in sales of the newspaper Clarín (1995-2012). An analysis from the political economy of communication). Razón y Palabra, 18(84).

Retegui, L. (2020). Métricas y cuantificación del rendimiento individual de los periodistas (Metrics and the quantification of individual journalistic performance: a study into the heart of a newsroom). Austral Comunicación, 9(1), 45-67. https://doi.org/10.26422/aucom.2020.0901.ret

Robledo-Dioses, K. \& Atarama-Rojas, T. (2018). Periodismo transmedia y consumo mediático de la generación millennials (Transmedia journalism and media consumption of the millennial generation). Anagramas, 17(33), 105-127. https://doi.org/10.22395/angr.v17n33a5

Rojas, H. (2006). Comunicación, participación y democracia (Communication, participation and democracy). Universitas, (62), 109-142. http://www.scielo.org.co/scielo. php?pid=S0120-48072006000200006\&script=sci_arttext\&tlng=en

Rothmann, W. \& Koch, J. (2014). Creativity in strategic lock-ins: The newspaper industry and the digital revolution. Technological forecasting and social change, 83, 66-83. https://doi.org/10.1016/j.techfore.2013.03.005

Sarrimo, C. (2017). The press crisis and its impact on Swedish arts journalism: Autonomy loss, a shifting paradigm and a "journalistification" of the profession. Journalism: Theory, Practice \& Criticism, 18(6), 664-679. https://doi.org/10.1177/1464884915625629

Schlesinger, P. \& Doyle, G. (2015). From organizational crisis to multi-platform salvation? Creative destruction and the recomposition of news media. Journalism, 16(3), 305-323. https://doi.org/10.1177/1464884914530223

Schmitz Weiss, A., de Macedo Higgins Joyce, V., Harlow, S., \& Alves, R. C. (2018). Innovation and Sustainability: A Relationship Examined among Latin American Entrepreneurial News Organizations. Cuadernos. info, (42), 87-100. https://doi.org/10.7764/cdi.42.1266

Schudson, M. (2010). News in crisis in the United States: panic-and beyond. In D. A. Levy \& R. Klein Nielsen (Eds.), The changing business of journalism and its implications for democracy (pp. 95-106). Oxford, UK: Reuters Institute. Retrieved from https://reutersinstitute. politics.ox.ac.uk/sites/default/files/2017-11/The\%20Changing\%20Business\%20of\%20 Journalism\%20and\%20its\%20Implications\%20for\%20Democracy.pdf\#page=101 
Siapera, E., Papadopoulou, L., \& Archontakis, F. (2015). Post-Crisis Journalism: Critique and renewal in Greek journalism. Journalism Studies, 16(3), 449-465. https://doi.org/10.1080/1461670X.2014.916479

Siles, I. \& Boczkowski, P. J. (2012). Making sense of the newspaper crisis: A critical assessment of existing research and an agenda for future work. New Media and Society, 14(8), 1375-1394. https://doi.org/10.1177/1461444812455148

SINCA. (s. f.). Circulación neta diaria (4 principales diarios y resto de los diarios) (Daily net circulation (4 main newspapers and the rest of the newspapers)) (data set). Retrieved from https://www.sinca.gob.ar/DatosBasicosSeries.aspx?Id=77

Starbird, K. (2017). Examining the alternative media ecosystem through the production of alternative narratives of mass shooting events on Twitter. In Proceedings of the AAAI Conference on Web and Social Media 2017 (pp. 230-239). AAII. https://www.aaai.org/ocs/ index.php/ICWSM/ICWSM17/paper/viewPaper/15603

Starke, C., Naab, T. K., \& Scherer, H. (2016). Free to Expose Corruption: The Impact of Media Freedom, Internet Access and Governmental Online Service Delivery on Corruption. International Journal of Communication, 10, 4702-4722. Retrieved from http://ijoc.org/index.php/ijoc/article/view/5712

Thurman, N. (2018). Newspaper Consumption in the Mobile Age: Re-assessing multi-platform performance and market share using “time-spent”. Journalism Studies, 19(10), 1409-1429. https://doi.org/10.1080/1461670X.2017.1279028

Thurman, N. \& Fletcher, R. (2019). Has Digital Distribution Rejuvenated Readership?: Revisiting the age demographics of newspaper consumption. Journalism Studies, 20 (4), 542-562. https://doi.org/10.1080/1461670X.2017.1397532

Usher, N. (2015). Newsroom moves and the newspaper crisis evaluated: space, place, and cultural meaning. Media Culture \& Society, 37(7), 1005-1021. https://doi.org/10.1177/0163443715591668

van der Burg, M. \& Van den Bulck, H. (2017). Why are traditional newspaper publishers still surviving in the digital era? The impact of long-term trends on the Flemish newspaper industry's financing, 1990-2014. Journal of Media Business Studies, 14(2), 82-115. https://doi.org/10.1080/16522354.2017.1290024

Verón, E. (2013). La semiosis social, 2: ideas, momentos, interpretantes (Social semiosis, 2: ideas, moments, interpreters). Barcelona, Spain: Paidós.

Víctima de la crisis en Brasil, cerró el periódico Jornal do Commercio (Victim of the crisis in Brazil, the newspaper Jornal do Commercio closed). (2016, April 30). Infobae. Retrieved from https://www.infobae.com

Villi, M. \& Hayashi, K. (2017). “The Mission is to Keep this Industry Intact”: Digital transition in the Japanese newspaper industry. Journalism Studies, 18(8), 960-977. https://doi.org/10.1080/1461670X.2015.1110499

Wadbring, I. \& Bergström, A. (2017). A Print Crisis or a Local Crisis?: Local news use over three decades.Journalism Studies, 18(2), 175-190. https://doi.org/10.1080/1461670X.2015.1042988

Wahl-Jorgensen, K. (2018). Challenging presentism in journalism studies: An emotional life history approach to understanding the lived experience of journalists. Journalism, 20(5), 670-678. https://doi.org/10.1177/1464884918760670 
Waisbord, S. (2017). Crisis y posprofesionalismo en el periodismo contemporáneo (Crisis and post-professionalism in contemporary journalism). In E. Miltchensein \& P. Boczkowski (Comps)., Titulares, hashtags y videojuegos. La comunicación en la era digital (Headlines, hashtags and video games. Communication in the digital age) (pp. 17-34). Buenos Aires: Manantial.

Waisbord, S. (2019). Communication: A Post-Discipline. Cambridge: Polity Press.

Zuluaga Trujillo, J. \& Gómez Montero, S. (2019). Medios nativos digitales en América Latina: agenda, sostenimiento e influencia (Digital Native Media in Latin America: Agenda, Sustainability and Influence). Chasqui. Revista Latinoamericana de Comunicación, 1(141), 301-316. https://doi.org/10.16921/chasqui.v0i141.3333

\section{SOBRE LOS AUTORES}

FACUNDO SUENZO, estudiante de doctorado en Medios, Tecnología y Sociedad en la Universidad de Northwestern (EE.UU.), donde también es afiliado al Center for Latinx Digital Media.

(iD) https://orcid.org/0000-0002-4757-1850

PABLo J. BoCzKowsKI, Hamad Bin Khalifa Al-Thani Professor en el Departmento de Estudios de la Comunicación de Northwestern University (EE.UU.).

(iD) https://orcid.org/0000-0002-9446-8303

EUGENIA MITCHELSTEIN, profesora asociada en la Universidad de San Andrés.

(iD) https://orcid.org/0000-0001-7355-8740 